\title{
Low-Dose Quetiapine Induced or Worsened Mania in the Context of Possible Undertreatment
}

\author{
Hun Y. Millard, MD, MA, Barbara A. Wilson, MD, and Douglas L. Noordsy, MD
}

Bipolar disorder is a mental illness with a lifetime prevalence of $2 \%$ and has a dramatic impact on quality of life. Mania is a distinct period of abnormal and sustained elevated, expansive, or irritable mood and increase in goal-directed activity or energy that lasts at least 1 week and is present for most of each day. Quetiapine is an atypical antipsychotic approved for the treatment of bipolar depression and mania. For the treatment of acute mania, a dose of 600 to $800 \mathrm{mg} /$ day is recommended. There has been concern of potential induction or worsening of hypomanic or manic symptoms at low doses via the ratio of 5HT2A/D2 receptor antagonism, which at lower doses favors greater 5HT2A receptor blockade and thus increases dopamine concentrations. This article describes a case report of hypomania worsening to mania with psychotic features in a drug-naive patient who was started on low-dose quetiapine. This case adds to the existing literature of case reports indicating that low-dose quetiapine may be associated with induction or worsening of hypomanic/manic symptoms, while acknowledging the difficulty of suggesting a causal relationship. (J Am Board Fam Med 2015;28:154-158.)

Keywords: Bipolar Disorder, Mental Health, Pharmacotherapy, Psychiatry, Quetiapine

Quetiapine is a second-generation dibenzothiazepine antipsychotic approved for the treatment of schizophrenia, major depression, bipolar disorder, bipolar depression, and mania. Off-label use for a variety of symptoms such as insomnia and agitation is also common. For the treatment of acute mania, quetiapine is titrated upward fairly quickly to 600 to $800 \mathrm{mg} /$ day. ${ }^{1,2}$ For treatment of bipolar depression, randomized controlled trials (RCTs) showed significant improvement in depressive symptoms at both 300- and 600-mg doses. ${ }^{1,3}$ At lower doses of quetiapine, rare case reports suggest concern about potential induction or worsening of hypomania or manic symptoms. ${ }^{4-13}$

Quetiapine is a dopamine, serotonin (5-hydroxytryptamine $[5 \mathrm{HT}])$, and norepinephrine blocker and has

This article was externally peer reviewed.

Submitted 26 March 2014; revised 18 July 2014; accepted 22 July 2014.

From the Department of Psychiatry, Geisel School of Medicine, Dartmouth University, Dartmouth-Hitchcock Medical Center, Lebanon, NH.

Funding: none.

Conflict of interest: none declared.

Corresponding author: Hun Y. Millard, MD, Yale Child Study Center, Yale School of Medicine, Yale University, 230 S. Frontage Road, New Haven, CT 06519 (E-mail: hun.millard@yale.edu). antihistaminergic properties. At 25 to $50 \mathrm{mg}$, quetiapine acts primarily as a histamine and $\alpha_{1}$ adrenergic antagonist, explaining its hypnotic effects, including daytime sedation. ${ }^{14}$ As concentrations increase, quetiapine activates the adrenergic system and antagonizes serotonin $5 \mathrm{HT}_{2 \mathrm{~A}}$ receptors; at higher concentrations it blocks $\mathrm{D}_{2}$ receptors. Serotonin acts on $\gamma$-aminobutyric acid neurons, inhibiting the release of dopamine and norepinephrine; thus, when serotonin $5 \mathrm{HT}_{2 \mathrm{~A}}$ receptors are blocked, serotonin is suppressed and dopamine concentrations are enhanced in the forebrain. ${ }^{15,16}$ At doses of $\leq 300 \mathrm{mg}$ of quetiapine, the ratio of $5 \mathrm{HT}_{2 \mathrm{~A}}$ to $\mathrm{D}_{2}$ receptor antagonism favors greater $5 \mathrm{HT}_{2 \mathrm{~A}}$ receptor blockade; consequently, serotonin $5 \mathrm{HT}_{2 \mathrm{~A}}$ receptors are blocked, but $\mathrm{D}_{2}$ receptors have not been significantly blocked at this dose, potentially leading to net pro-dopaminergic effects.

\section{Case Review}

Mr. B is a 38-year-old married white man who presented to his local community mental health center on an emergent basis, reporting increased irritability, anxiety, grandiosity, excessive spending, as well as increased goal-directed activity and a 
decreased need for sleep over the past month. Because an evaluation with a psychiatrist could not be scheduled promptly, he contacted his primary care physician (PCP) and asked for pharmacologic intervention. Mr. B reported a history of similar episodes occurring cyclically every few years since his early 20s. However, he had never been diagnosed with a mental illness or hospitalized, nor had he ever taken psychiatric medications.

Over the phone, Mr. B's PCP prescribed quetiapine (Seroquel) $25 \mathrm{mg}$ at bedtime (QHS), with a plan to increase to $25 \mathrm{mg}$ in the morning and $50 \mathrm{mg}$ QHS. A meeting with a licensed clinical mental health counselor the next day also was arranged by his PCP. Mr. B met with the licensed clinical mental health counselor and 13 days later saw his PCP for a follow-up appointment; he was noted to be "hypomanic" with symptoms of irritability, poor sleep, distractibility, and increased talking and spending. The plan was for Mr. B to increase quetiapine to $50 \mathrm{mg}$ twice daily for 3 days, then increase by $50 \mathrm{mg}$ every 3 days until he reached 100 $\mathrm{mg}$ twice daily.

Approximately 1 month later, Mr. B contacted his PCP's office and stated "things have gotten worse." Previous complaints continued but now he felt more agitated. He consistently took quetiapine daily but only $25 \mathrm{mg}$ in the morning and $50 \mathrm{mg}$ QHS, complaining of excessive sedation at higher doses. After approximately 6 weeks on quetiapine, he left his home one evening, feeling that his presence was unsafe for his family, although he himself did not have any concern of harming others. The following day Mr. B checked into a hotel and had for the first time a distinct delusion in which he believed he was "Jesus." Later that evening he began to have auditory hallucinations and rather suddenly believed an elderly stranger in the hotel hallway was the "devil" trying to harm him and his family, and he killed her.

Mr. B had no criminal or violent history and had not reported prior psychotic symptoms. When he arrived at jail, quetiapine was discontinued and he was started on chlorpromazine to control his insomnia and psychosis. Approximately 1 month later he was no longer psychotic and noted to be calm and depressed. After stabilization, chlorpromazine was eventually stopped. He remains on lithium monotherapy. He is currently awaiting trial, at which he intends to submit a plea of not guilty by reason of insanity.

\section{Review of the Literature}

There are 13 existing case reports in the literature documenting the tempo of hypomanic and manic symptoms (and resolution of symptoms) that coincide with the onset and discontinuation of low-dose quetiapine. The theory that the $5 \mathrm{HT}_{2 \mathrm{~A}}$-to- $\mathrm{D}_{2}$ receptor antagonism ratio, which at lower doses favors $5 \mathrm{HT}_{2 \mathrm{~A}}$, induces hypomanic/manic symptoms is the most popular. ${ }^{4-13}$ However, there are additional hypotheses as well. Lykouras et $\mathrm{al}^{10}$ and Michalopoulou and Lykouras ${ }^{16}$ suggest that loose binding of $\mathrm{D}_{2}$ receptors may cause fast dissociation from receptors and thus increase the overall transmission of dopamine. Not only is quetiapine itself a partial agonist of $5 \mathrm{HT}_{1 \mathrm{~A}}$ receptors, increasing dopamine release, the simultaneous $5 \mathrm{HT}_{2 \mathrm{~A}} / \mathrm{D}_{2}$ receptor blockade also produces $5 \mathrm{HT}_{1 \mathrm{~A}}$ receptor activation. ${ }^{9}$ There is also the theory that quetiapine can induce or worsen manic symptoms because of antidepressant effects via the inhibition of norepinephrine reuptake transporters. ${ }^{8}$ Another possible contributor to explain mania induction is the unique disposition of the patient via the CYP450 metabolism process. Quetiapine is metabolized by the CYP450-3A4 isoenzyme; those who are poor metabolizers may not benefit from quetiapine unless they receive higher doses. ${ }^{5,9}$ It is worth noting that the only RCT evaluating the antidepressant effects of quetiapine in bipolar I and II depression (supported by funding from AstraZeneca, the maker of quetiapine) reports that the incidence of treatment-emergent mania was equivalent to placebo. $^{2}$

\section{Discussion}

We present this case because it highlights several realities in mental health treatment, as well as areas of clinical consideration. PCPs are often at the front lines of mental health care, treating people with complex presentations, because patients often do not have ready access to psychiatrists. Mr. B was disenchanted with the community mental health system and sought help from his PCP, who assessed him as hypomanic. Unless there are psychotic symptoms or an imminent need for hospitalization, the diagnosis of hypomania versus mania is often equivocal for physi- 
Table 1. Diagnostic and Statistical Manual of Mental Disorders Criteria for Diagnosis of a Hypomanic and Manic Episode

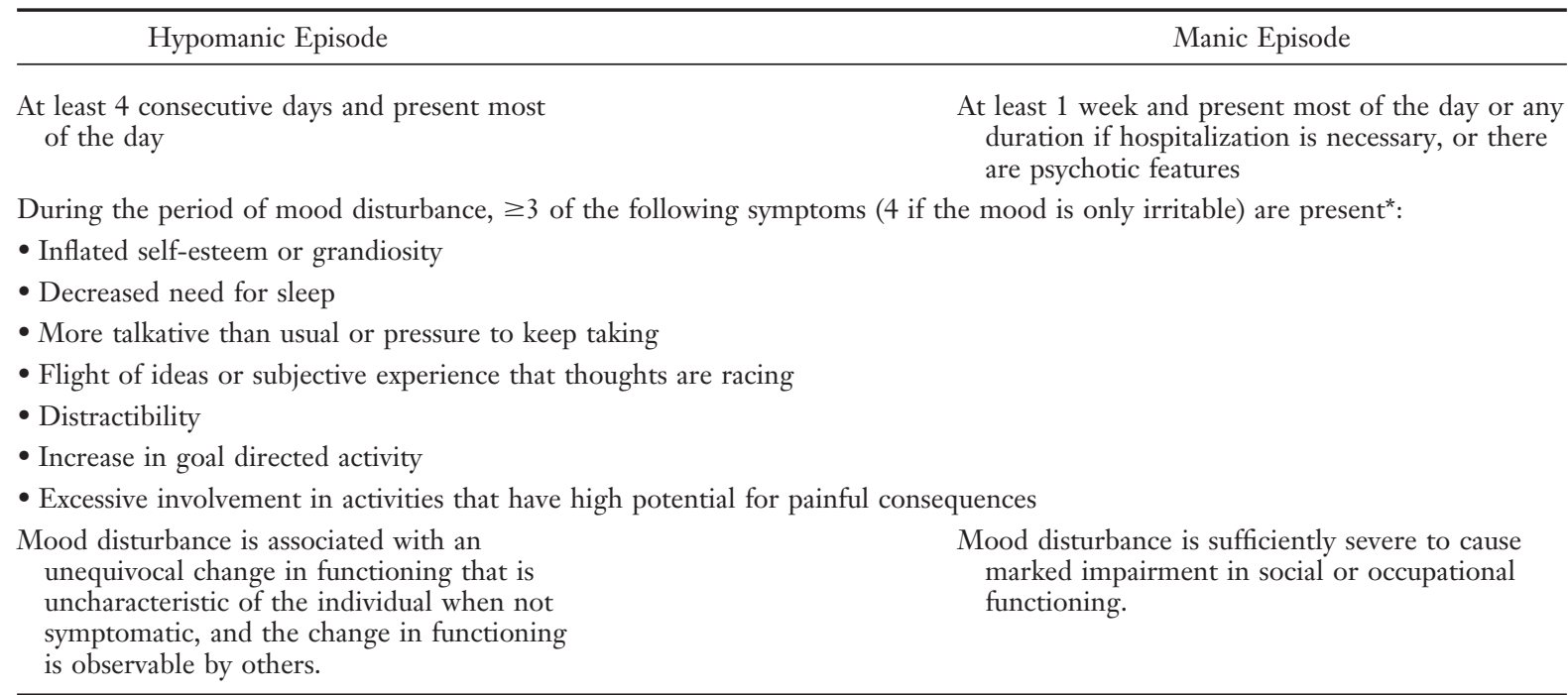

*These represent a noticeable change from usual behavior.

cians who do not routinely monitor patients with bipolar disorder. Symptom criteria for hypomania and mania are the same, except for psychotic features and assessment of the overall level of impairment, including the need for hospitalization, as shown in Table 1. Hypomanic symptoms are less severe and impairing, yet symptoms must still be observable by others and represent a change from the individual's baseline. ${ }^{17}$

Multiple first-line medications are used for the treatment of mania; lithium, valproate, and secondgeneration antipsychotics have the greatest evidence of efficacy. The majority of RCTs of bipolar disorder have included patients with bipolar I disorder, and evidence in bipolar II disorder is limited; thus treatments for hypomania are analogues to that of mania. If single-agent treatment is not effective in improving symptoms within a short period, a second agent may be added or the patient may be switched to an alternative first-line medication. $^{18}$

Given Mr. B's history of cyclic mood disturbances, the PCP correctly chose a sedating antipsychotic to tackle Mr. B's insomnia, irritability, and anxiety. The physician used the "low and slow" tactic in titrating quetiapine, which is often helpful for monitoring side effects as well as promoting medication compliance. In this case, however, lowdose quetiapine may have precipitated the worsening of symptoms or simply was ineffective in slowing an already evolving manic episode. A diagnosis of mania, rather than hypomania, may have fostered more aggressive titration and dosing of quetiapine and greater urgency for follow up. Mr. B did not follow the full titration because of intolerable side effects, opting instead to stay at less than half the dose recommended by his PCP and 8 times lower than the lowest recommended dose for acute mania (quetiapine $600 \mathrm{mg}$ ). ${ }^{14}$

The case of Mr. B is puzzling. There are different possible conclusions that can be drawn from his presentation and outcome. First, there could be a causal relationship between low-dose quetiapine and the escalation of mania. ${ }^{4-13}$ Mr. B's manic symptoms subsided and eventually remitted after discontinuing quetiapine and switching to chlorpromazine. The relationship between manic symptoms and quetiapine would be stronger if we were certain of a diminution in manic symptoms before chlorpromazine was initiated. Second, Mr. B may be a nonresponder or underresponder to quetiapine at any dose, and another mood stabilizer would simply have been more effective. Finally, Mr. B's quetiapine dose may have been subtherapeutic and his mania progressed because he was not taking the recommended daily dose ( $\geq 600 \mathrm{mg}$ for acute mania or $\geq 300 \mathrm{mg}$ for bipolar depression). ${ }^{1}$

\section{Conclusion}

While Mr. B's symptoms were severe and do not represent the majority of patients who suffer from 
bipolar disorder, the complexities and uncertainties of managing mental illness in the outpatient setting (represented here by unclear initial presentation, side effects of medications, patient underadherence, and difficulties with communication) are commonplace. When using quetiapine-or any other second-generation antipsychotic medication-in situations like Mr. B's, we make the following suggestions:

- Despite case reports positing a causal relationship between the induction or escalation of manic symptoms while taking quetiapine at various doses, existing RCTs fail to statistically confirm such an effect. ${ }^{2,19}$ An adequate dose of quetiapine should still be considered as a treatment option for acute hypomania or mania, as well as monotherapy for bipolar depression.

- Mid-dose quetiapine (300 mg) is recommended for bipolar depression and as adjunctive therapy for unipolar depression. ${ }^{14}$ Quetiapine is FDAapproved as monotherapy for bipolar depression.

- High-dose quetiapine (600 to $800 \mathrm{mg}$ ) is the recommended dose for acute mania or psychosis. ${ }^{14}$ Quetiapine is FDA-approved for acute schizophrenia and mania.

- We agree with the findings of Cordoba et $\mathrm{al}^{7}$ that being more aggressive in titrating quetiapine may be most beneficial to the already hypomanic/ manic patient.

- If symptoms continue to worsen while taking a specific mood stabilizer after therapeutic dosing has been reached, then a second agent should be added or an alternative mood stabilizer should be considered because there may be individual differences in medication response.

- Antidepressants may precipitate or worsen mania and thus are typically discontinued in the treatment of acute mania. ${ }^{18}$

- Closer follow-up through brief medication checks is advised so symptoms can be monitored, side effects discussed, and the need for a higher level of care assessed at regular intervals.
- Psychoeducation about illness, recognition of symptoms, and medication adherence is an important component of treatment and relapse prevention. ${ }^{18}$

The authors thank Dr. Matthew S. Duncan for his support and Heather B. Blunt for library services.

\section{References}

1. Vieta E. Mood stabilization in the treatment of bipolar disorder: focus on quetiapine. Hum Psychopharmacol 2005;20:225-36.

2. Vieta E, Calabrese JR, Goikolea JM, Raines S, Macfadden $W$; BOLDER Study Group. Quetiapine monotherapy in the treatment of patients with bipolar I or II depression and a rapid-cycling disease course: a randomized, double-blind, placebo-controlled study. Bipolar Disord 2007;9:413-25.

3. Calabrese JR, Elhaj O, Gajwani P, Gao K. Clinical highlights in bipolar depression: focus on atypical antipsychotics. J Clin Psychiatry 2005;66(Suppl 5): 26-33.

4. Biancosino B, Marmai L, Facchi A, Rossi E, Grassi L. Quetiapine may induce mania: a case report. Can J Psychiatry 2003;48:349-50.

5. Erberk-Ozen N. Manic symptoms probably associated with short-term low-dose quetiapine use. Adv Ther 2008;25:53-8.

6. Benazzi F. Quetiapine-associated hypomania in a woman with schizoaffective disorder. Can J Psychiatry 2001;46:182-3.

7. Cordoba S, de Asis J, Kotbi N, Mahgoub N. Worsening mania associated with slow increase of quetiapine dose. J Neuropsychiatry Clin Neurosci 2011;23: E5-6.

8. Gnanavel S. Quetiapine-induced manic episode: a paradox for contemplation. BMJ Case Rep 2013; 2013. pii:bcr2013201761.

9. Khalil RB, Baddoura C. Quetiapine induced hypomania: a case report and a review of the literature. Curr Drug Saf 2012;7:250-3.

10. Lykouras L, Oulis P, Hatzimanolis J. Manic symptoms associated with quetiapine treatment. Eur Neuropsychopharmacol 2003;13:135-6.

11. Mishra A, Moore PB, Hobbs R. Does quetiapine have mood altering properties? J Psychopharmacol 2004;18:281-4.

12. Pacchiarotti I, Manfredi G, Kotzalidis GD, Girardi P, Tatarelli R. Quetiapine-induced mania. Aust N Z J Psychiatry 2003;37:626.

13. Atmaca M, Kuloglu M, Buyukbayram A, Tezcan E. Quetiapine-associated and dose-related hypomania in a woman with schizophrenia. Eur Psychiatry 2002;17:292-3.

14. Stahl S. Stahl's essential psychopharmacology: neuroscientific basis and practical application. 
4th ed. Cambridge: Cambridge University Press; 2013.

15. Rachid F, Bertschy G, Bondolfi G, Aubry JM. Possible induction of mania or hypomania by atypical antipsychotics: an updated review of reported cases. J Clin Psychiatry 2004;65:1537-45.

16. Michalopoulou PG, Lykouras L. Manic/hypomanic symptoms induced by atypical antipsychotics: a review of the reported cases. Prog Neuropsychopharmacol Biol Psychiatry 2006;30:549-64.
17. Diagnostic and Statistical Manual of Mental Disorders. 5th ed. Washington, DC: American Psychiatric Association; 2013.

18. Stern TA, Rosenbaum JF, Fava M, Biederman J, Rauch S. Massachusetts General Hospital comprehensive clinical psychiatry. Philadelphia: Mosby; 2008.

19. Benyamina A, Samalin L. Atypical antipsychoticinduced mania/hypomania: a review of recent case reports and clinical studies. Int J Psychiatry Clin Pract 2012;16:2-7. 